CRÍTICA, Revista Hispanoamericana de Filosofia

Vol. XXX, No. 90 (diciembre 1998): 3-21

\title{
ON THE UTILITY OF GLOBAL SUPERVENIENCE*
}

\author{
Manuel Pérez Otero \\ Departamento de Lógica, Historia y Filosofía de la Ciencia \\ Universidad de Barcelona
}

\section{Introduction}

The relation of supervenience is a kind of dependence between properties or, more exactly, families of properties. That dependence would be roughly stated in the dictum "no difference in supervenient properties without difference in subvenient properties". In other words, if A and B are families of properties and the properties in A supervene on the properties in $\mathrm{B}$, then there cannot be two objects having the same B-properties but differing regarding some A-property.

In fact, there is not just one but several non-equivalent relations of supervenience. The vague characterization we have mentioned corresponds approximately to the notion of strong supervenience put forward by Jaegwon Kim, the

* I would like to thank Josep Corbí and Manuel García-Carpintero for their useful comments and suggestions about the topic discussed in this paper. Financial support has been provided by the research projects PB96-1091-C03-03, funded by the Ministry of Education and Culture of Spain, and 1997SGR 00396, funded by the Generalitat of Catalunya. 
author who has contributed the most to the important position occupied by the concept of supervenience in recent metaphysics and philosophy of mind. Kim has stressed one of the main philosophical motivations for paying attention to such a notion: the holding of a supervenience relation between two classes of properties can be understood as a metaphysical dependence of the supervenient on the subvenient properties, without implying that there is also a stronger and more controversial relation of reduction between the two kinds of properties.

Nevertheless, even that strong supervenience relations turns out to be too demanding. Sometimes the A-properties an object $\mathrm{x}$ can have depends on a class of B-properties possessed not just by $\mathrm{x}$ itself but by the objects that constitute the (physical, historical, spatial, ...) context in which $\mathrm{x}$ is located. The notion of global supervenience was proposed to pick out that idea of dependence or determination by the context. In that way, global supervenience appears to be a relation of dependence weaker than strong supervenience and some philosophers think the concept of global supervenience is apt to formulate weak claims of dependence between families of properties. The status and utility of that concept, however, has been disputed by Kim, who has suggested why the real difference between strong and global supervenience is not so interesting. I want to point out some traits of global supervenience, partly related to Kim's remarks about its relationship with strong supervenience, which make it a little clearer why it is a not very useful notion. In section I precise definitions of strong and global supervenience are presented, and some reasons are given to think that global supervenience does not quite satisfy the philosophical motivation that engendered it. In section II Petrie's and Paull and Sider's counterexamples to the equivalence of global and strong supervenience are 
discussed, and a weak thesis of equivalence is put forward.

\section{I}

When a supervenience relation holds there is no difference in supervenient properties without a corresponding difference in subvenient properties. In order to capture one of the senses of that idea, Kim proposed the notion of strong supervenience defined as follows. Assuming that A and B are two families of properties closed under the Boolean operations of complementation, conjunction and disjunction.

A strongly supervenes on B just in case, necessarily, for each $\mathrm{x}$ and each property $\mathrm{F}$ in $\mathrm{A}$, if $\mathrm{x}$ has $\mathrm{F}$, then there is a property $G$ in $B$ such that $x$ has $G$, and necessarily if any y has $\mathrm{G}$, it has $\mathrm{F}$.

Kim (1987) quotes a characterization due to Brian McLaughlin which is similar to his and which does not place any restriction on $\mathrm{A}$ and $\mathrm{B}$ :

The family of properties A strongly supervenes on the family of properties B iff for any worlds $\mathrm{v}$ and $\mathrm{w}$, and for any objects $\mathrm{x}$ and $\mathrm{y}$, if $\mathrm{x}$ has in $\mathrm{v}$ the same B-properties (the same properties in B) that $\mathrm{y}$ has in $\mathrm{w}$, then $\mathrm{x}$ has in $\mathrm{v}$ the same A-properties that $\mathrm{y}$ has in $\mathrm{w}^{2}$

We will assume this second, possible-worlds definition of "strong supervenience" since it is more general than (and consequently also non-equivalent to) the former, modaloperator definition. ${ }^{3}$

${ }^{1}$ See Kim (1984), p. 65 and Kim (1987), pp. 79-80.

${ }^{2}$ See Kim (1987), p. 81.

${ }^{3}$ Some objections to the identification of the two formulations of strong supervenience are given in McLaughlin (1995), pp. 25-30. I disagree, however, with one of them. According to McLaughlin some 
When the properties in the base set (that is, the set of subvenient properties) are the more fundamental physical properties or the more basic ones from among the properties postulated by current physics, the claim that every set or family of properties strongly supervenes on them has been regarded as a suitable formulation of physicalism. Another theoretical purpose served by strong supervenience (closely related to the issue of physicalism) is this: the strong supervenience of a particular family of properties A on physical properties appears sometimes as a proper requirement for the causal efficacy of the properties in A (causal efficacy that, according to some philosophers, is, in turn, a necessary condition for such so-called properties to be properties at all).

The properties whose supervenient nature we inquire into are in many cases properties expressed or referred to by a predicative expression of ordinary language (for instance, moral, mental or aesthetic properties). Whether one of these properties is intrinsic or extrinsic is something we do not know beforehand; therefore it would not be reasonable to use a characterization of strong supervenience which excluded extrinsic or relational properties from the families of properties apt to be considered supervenient on others (simply because we would not even know if it makes sense to ask about the supervenience on something

uses of "necessarily" cannot be captured by quantification over possible worlds. For instance, there are metaphysically necessary truths (Kripkean examples like "water is $\mathrm{H}_{2} \mathrm{O}$ ") that are not analytically necessary truths, but, he says, the class of metaphysically possible worlds is the same as the class of analytically possible worlds (see McLaughlin (1995), pp. 26-27). I share the Kripkean view that McLaughlin adopts about the existence of necessary non-analytical truths; but I think that if we decide to speak of the analytically possible worlds, every reason to differentiate metaphysical necessity from analitical necessity is also a reason for not identifying the analytically possible worlds with the metaphysically possible worlds. 
else of one of those properties not overtly intrinsic nor overtly extrinsic). ${ }^{4}$ This being the case, there is little point in excluding extrinsic or relational properties from the set of purportedly supervenient properties.

But the situation is different regarding the properties in the base set. To postulate and detect physical properties intrinsic to their bearers seems to be a desideratum of the more fundamental physical theories. Thus, it has been implicitly assumed that interesting claims of strong supervenience on physical properties must be taken with a restriction on the range of properties that are suited to form the base set: only purely intrinsic properties can be counted as subvenient properties.

Such an assumption, whether or not there is enough justification for it, is shown, for instance, by the fact that Putnam's Twin Earth case appears as a prima facie counterexample to the strong supervenience of mental properties on physical properties. Let us recall very briefly what the example is. Oscar, an inhabitant of Earth, has a physically identical counterpart, Twin Oscar, who lives in Twin Earth. This is a planet very much like Earth except that there is not water in it but twater, a substance observationally indiscernible from water, but whose chemical structure is not $\mathrm{H}_{2} \mathrm{O}$. Therefore, and assuming externalism in the individuation of mental states (in fact, Putnam's example was originally designed to defend the externalism of meaning), Oscar's thoughts about water are not shared by

4 I am relying on an intuitive distinction between, on the one hand, intrinsic properties, and, on the other hand, and without discriminating between them, extrinsic or relational properties. This distinction is worked out, for instance, in Lewis (1983), pp. 355-358. The problem I will put forward is, I think, relatively independent of how we elucidate the intrinsic/extrinsic distinction. Therefore, I will proceed without trying to characterize that distinction. 
Twin Oscar, whose thoughts are about twater. ${ }^{5}$ Now if we allowed ourselves to put extrinsic or relational properties into the physical characterization of Oscar and Twin Oscar, they would be not only mentally but also physically different. That shows that insofar as the Twin Earth cases are thought as being in opposition to the strong supervenience of the mental on the physical, it is implicitly assumed that extrinsic or relational properties are excluded from the set of subvenient properties.

The physicalist requirement for some family of properties A to strongly supervene on physical properties proves to be quite demanding, if we understand it in such a way (i.e., if we count only physical properties which are intrinsic in the base set). ${ }^{6}$ It so happens that a lot of properties do not satisfy this condition, that is, they do not depend on the intrinsic physical traits of their instances, even if they very probably depend on the physical traits of the context where their instances are located. We would like to say that these properties (including, maybe, mental properties, if Twin Earth arguments make an impression on us), nonetheless, are not something over and above the physical world.

And this is where global supervenience comes in. Kim introduced the concept in Kim (1984). Let A and B be families of properties

5 See Putnam (1975). Burge has also argued for the dependence of beliefs on the believer's context (see, for instance, Burge (1979)). But in García-Carpintero (1994) there is a defense of the strong supervenience of the mental on intrinsic physical properties without giving up either the causal efficacy of mental properties or the externalism in the individuation of mental content presupposed in the Twin Earth argument.

6 It is quite demanding even if, such as it has been defined, strong supervenience only expresses mere covariance between supervenient and subvenient properties but, as Kim has pointed out, it falls short of fully capturing intuitive traits of the relation of dependence, like its asymmetric character (see Kim (1987), pp. 139-149). 
A globally supervenes on B iff worlds that are B-indiscernible are also A-indiscernible. ${ }^{7}$

The definition of indiscernibility used was this:

Two worlds are indiscernible with respect to A (A-indiscernible) iff for every property $\mathrm{F}$ in $\mathrm{A}$ and every individual $\mathrm{x}, \mathrm{x}$ has $\mathrm{F}$ in one just in case $\mathrm{x}$ has $\mathrm{F}$ in the other.

Kim's characterization of "indiscernibility" has a number of drawbacks. ${ }^{8}$ Maybe the more prominent of them is its inapplicability to worlds not related by transworld identity relations between individuals in their domains; therefore, it is of no use by those who claim that individuals are world-bounded. To overcome this difficulty we could employ a different characterization:

Two worlds $\mathrm{v}$ and $\mathrm{w}$ are A-indiscernible iff there is an isomorphism between the structure determined by the A-properties in the domain of $\mathrm{v}$ and the structure determined by the A-properties in the domain of $\mathrm{w}$ (i.e., there is a one-to-one function from the domain of $\mathrm{v}, \mathrm{U}$, onto the domain of $\mathrm{w}$ such that for every property $\mathrm{P}$ in $\mathrm{A}$, and for every e in $\mathrm{U}, \mathrm{P}(\mathrm{e})$ iff $\mathrm{P}(\mathrm{f}(\mathrm{e}))$ ).

The notion of global supervenience would provide us with some theoretical means to formulate thesis of deter-

${ }^{7}$ See Kim (1984), p. 68.

${ }^{8}$ McLaughlin has noted some of these drawbacks in McLaughlin (1995), pp. 31-34. There is another problem concerning the concept of global supervenience stemming from the aim to express relations of dependence in terms of indiscernibility between possible worlds: granting a certain view about possible worlds, for some set B of properties (the set of physical properties may be a good candidate) any set of properties will be considered globally supervenient on B; not for the intended reasons, however, but trivially by virtue of the fact that there are no two different B-indiscernible possible worlds. I know of no solution to this difficulty. 
mination or covariance weaker than those asserted using strong supervenience. We can, for instance, express the idea that properties whose instantiation depends on physical traits historically or spatially distant from their bearers are, in a weak but important sense, also determined by physics: they globally supervene on physical properties. Pairs of worlds like those suggested by the Twin Earth argument are not straightforward counterexamples to the global supervenience of mental properties on physical properties. This fact would require that Oscar's world and Twin Oscar's world were physically indiscernible (in the sense defined), and it is not clear at all that this is so.

To maintain homogeneity with regard to the notion of strong supervenience, it seems appropriate to read the definition of global supervenience with an implicit restriction on the base set: only intrinsic properties can be members of it. Hence, the very point of the concept of global supervenience appears to be this: we capture the idea that certain properties are dependent on or determined by extrinsic or relational traits of their instances by means of a notion of supervenience in which we stick to intrinsic properties in the base set, but which involves not only the properties possessed by the objects which instantiate the supervenient properties, but also the properties of the objects in their surroundings, the properties of the context.

Now an important problem arises with this picture. Let us consider the property of being between two green objects. That property, call it " $F$ ", has to be supervenient on physical traits of the whole context wherever it is instantiated. ${ }^{9}$ But it turns out that our present notion of global supervenience does not capture this seemingly obvious and

9 That holds clearly if we assume that colors supervene on physical properties. Those who are reluctant to accept this may use instead the property of being between two objects that are $G$, where $\mathrm{G}$ is any property they take to be supervenient on the physical. 
innocuous fact, that is, $\mathrm{F}$ does not globally supervene on physical properties. To see it we just have to consider the following worlds, $\mathrm{v}$ and $\mathrm{w}$ : the domain of $\mathrm{v}$ is composed by two green objects, a and c, and one blue object, b, located between them. In $w$ there are three objects, $a^{\prime}, b^{\prime}$ and $\mathrm{c}^{\prime}$ sharing with $\mathrm{a}, \mathrm{b}$ and $\mathrm{c}$, respectively, all intrinsic physical properties; but in $\mathrm{w}, \mathrm{c}^{\prime}$ is between $\mathrm{a}^{\prime}$ and $\mathrm{b}^{\prime}$ (if Kim's definition of indiscernibility is used, let $\mathrm{a}^{\prime}, \mathrm{b}^{\prime}$ and $\mathrm{c}^{\prime}$ be, respectively $\mathrm{a}, \mathrm{b}$ and $\mathrm{c}$ ). Worlds $\mathrm{v}$ and $\mathrm{w}$ are not $\mathrm{F}$ indiscernible because $\mathrm{b}$ in $\mathrm{v}$ is $\mathrm{F}$ but no object in $\mathrm{w}$ is $\mathrm{F}$; however, $\mathrm{v}$ and $\mathrm{w}$ are physically indiscernible as it is shown by the existence of the bijection which assigns $\mathrm{a}^{\prime}, \mathrm{b}^{\prime}$ and $\mathrm{c}^{\prime}$ to, respectively, a, b and c.

If extrinsic or relational properties are included in the base set, we will obtain the wanted global supervenience of $\mathrm{F}$ on the physical, since, for instance, the aforementioned worlds $\mathrm{v}$ and $\mathrm{w}$ will no longer be physically indiscernible: a certain relational spatial property, to be between two objects with such and such physical traits (the physical traits on which green supervenes), is instantiated by $\mathrm{b}$ in $\mathrm{v}$ but by no object in w. In general, it appears that to fully state the idea of determination by global context, reference must be done to extrinsic subvenient traits. This is hardly surprising regarding our property $\mathrm{F}$, because it is an overtly extrinsic one. But, we should remember, the extrinsic or intrinsic character of many other properties will not be so manifest. For them, therefore, the hypothesis that their global supervenience on physical properties is conditioned on the inclusion of extrinsic or relational properties on the base set will not be a trivial matter.

But such a need to take account of extrinsic properties virtually amounts to the failure of the notion of global su-

Manuel García-Carpintero provided me with the example in the main text. 
pervenience: if, accordingly, we also include in the set of strongly subvenient properties the extrinsic or relational ones, then F strongly supervenes on physical properties too, just for the reasons already mentioned (objects differing about having $\mathrm{F}$, like $\mathrm{b}$ and $\mathrm{b}^{\prime}$, also differ in relational physical properties). Following this line of reasoning, we might justifiably think in general that interesting cases of global supervenience will also be cases of strong supervenience (I am going to qualify this suggestion in section II). If that hypothesis were right then, for reasons of simplicity, we had better have available a single general notion of strong supervenience, with two specific versions depending on whether or not only intrinsic properties belong to the base set. To put it in a nutshell, there is little room for global supervenience because to do its work relational properties must be included in the set of subvenient properties and, in this case, this work maybe can be done by strong supervenience.

A similar idea can be drawn from some more recent comments by Kim against the utility of global supervenience. This passage illustrates the issue:

Examples like these [Kim is talking about properties such as the economic value of a coin or being the tallest man, to which we could add our property F], therefore, do not call for global supervenience. What they call for is an explicit recognition of relations and relational properties. The lesson we learn from the Putnam and Burge-style cases that is relevant to the topic of supervenience is that contrary to what used to be taken for granted, many intentional states turn out not to be "intrinsic" of "internal" to the subjects to whom they are attributed. Rather, they turn out to be "extrinsic" and "noninternal", dependent in complex ways on physical and social factors outside the subjects. It is no surprise then that we must seek a wider physical supervenience base for them, including relations and relational properties, if they are thought to be supervenient on the physical. A full 
account of these cases will be facilitated by a generalization of "strong" [...] supervenience to accommodate relations as well as properties. ${ }^{10}$

And, more explicitly, in a later writing he claims

[...] it seems a plausible conjecture that if extrinsic properties are included in both the supervenient and subvenient sets - in particular, if, along with the usual Boolean operations, identity and quantification are allowed for property composition - again the equivalence will obtain. ${ }^{11}$

This last quotation is extracted from a brief discussion on Petrie's and Paull and Sider's counterexamples to the equivalence between global and strong supervenience; I return to it in next section, where I address that question. Regarding the preceding quotation, the relevant and new point I want to stress is that this kind of "explicit recognition of relations and relational properties" that Kim talks about would already be needed in the formulation of global supervenience, as our example above (property F) has shown. Therefore, there is no choice (relatively to our aim to find a sense of "supervenience" which is appropriate for saying that being worth five dollars, being the tallest man, being between two green objects or having a thought about water are properties supervenient on physical properties) between resorting to global supervenience or generalizing strong supervenience with a recognition or relational properties, because such a recognition is necessary anyway.

II

I have just claimed there is a kind of equivalence between global and strong supervenience. In the line of Kim's last

${ }^{10}$ Kim (1987), pp. 88-89.

${ }^{11} \operatorname{Kim}(1993)$, p. 170. 
quotation, I have suggested that cases of global supervenience are likely to be cases of strong supervenience. Some qualifications are in order given that, in fact, the two notions are not equivalent. Global supervenience is weaker. So, in this section I will try to be a little more precise about the sense in which the two notions would be equivalent.

In Kim (1984), p. 69, a supposed proof of the equivalence was advanced. However, as some authors have pointed out and as Kim himself recognized later (Kim (1987), p. 82) there was a mistake in his derivation of strong supervenience from global supervenience. Bradford Petrie presented this counterexample: the set of supervenient properties A contains just the property $\mathrm{S}$ and the set of subvenient properties B contains just the property $\mathrm{P}$. Let $\mathrm{w}$ and $\mathrm{w}^{\prime}$ be two worlds, each with two individuals $\mathrm{x}$ and $\mathrm{y}$. In $\mathrm{w} \mathrm{x}$ has $\mathrm{S}$ and $\mathrm{P}$, and $\mathrm{y}$ has $\mathrm{P}$. In $\mathrm{w}^{\prime}$, $\mathrm{x}$ has $\mathrm{P}$ but not $\mathrm{S}$, and y lacks $\mathrm{P} .{ }^{12}$ This situation is perfectly consistent with the global supervenience of $\mathrm{A}$ on $\mathrm{B}$, but it rules out the corresponding strong supervenience.

Why then, if global supervenience does not entail strong supervenience, can the latter be used whenever we want to use global supervenience? The reason has two stages:

(i) First, the purely logical content of our metaphysical claims about supervenience is not the only part of them which interests us. We usually assume some background conditions about the existence and distribution of possible worlds. I will turn below to how these assumptions work.

(ii) Even granting those conditions, not every case of global supervenience between sets of properties is a case of strong supervenience (as another counterexample, coming from Paull and Sider, will show later). My suggestion, however, is that when the base set is given not by extension but by comprehension, in the way usual claims of super-

12 See Petrie (1987), p. 121. 
venience display (something supervenes on the physical or the biological properties, that is, families of properties that are, the families not the properties, in a sense, natural), and if we include extrinsic properties among these subvenient properties, then cases of global supervenience are also cases of strong supervenience.

My remarks about (i) are entirely inspired by the comments of Cranston Paull and Theodore Sider on Petrie's counterexample, although I disagree with some of their claims. ${ }^{13}$ Paull and Sider argue that Petrie has not proved that global supervenience does not entail strong supervenience because his counterexample is not enough to prove thesis $(\mathrm{P})$ :

(P) there are sets A and B such that A supervenes globally but not strongly on B.

Petrie's aforementioned set A does not supervene strongly on his set $\mathrm{B}$, but to guarantee the global supervenience we have to take into account possible worlds beyond the pair of worlds considered by Petrie:

[G]lobal supervenience is a universally quantified thesis [...] Although the small portion of logical space Petrie considers gives us a counterexample to the strong supervenience of $\mathrm{A}$ and $\mathrm{B}$ without yet giving us a counterexample to their global supervenience, his proof is not finished. We need some reason to think that the remainder of logical space will not provide a counterexample to the global supervenience of A on $\mathrm{B}^{14}$

Petrie's case certainly does not serve to prove $(\mathrm{P})$. But I think Paull and Sider are mistaken in their suggestion that the non-entailment of strong supervenience by global

13 See Paull et al. (1992), pp. 835-840.

14 Ibid., p. 837. 
supervenience implies $(\mathrm{P})$ and also when they say "apparently it is $(\mathrm{P})$ that Petrie is trying to establish with his case" (Paull et al. (1992), p. 837). What Petrie seeks, and indeed achieves, is

[...] to construct an example which shows that there is no inconsistency in supposing that global supervenience holds in some cases in which strong supervenience does not. ${ }^{15}$

And that is what is required to refute the thesis that global supervenience entails strong supervenience, because it is the logical equivalence between global and strong supervenience that is at issue. To express it with an analogy: suppose we ask whether thesis (a) "there exist ravens" implies or not thesis (b) "there exist black ravens". To prove that (a) does not imply (b) we do not have to prove that there are ravens which are not black. In its more natural reading, it is a question about the relations of logical consequence between (a) and (b). It would be misleading to state that just because every raven is black, or, even, in all possible worlds every raven is black, theses (a) and (b) turn out to be equivalent.

In the same way, the thesis of the equivalence of global and strong supervenience, which Kim erroneously advanced, has a preferred reading to which Petrie's counts as a proper counterexample, even if it falls short of entailing (P). The most definite evidence for it, in my view, is to be found in what Kim himself took the putative equivalence to mean, which is manifest in how he aimed to prove it: he tried to derive strong supervenience from global supervenience in a logical, purely formal way. Just a small mistake found its way in.

In spite of all this, what I take as the main thrust in Paull and Sider's reasoning is correct and worthy: anyone

15 Petrie (1987), p. 121. (My emphasis). 
who wants to use global supervenience to state dependence relations weaker than those supported by strong supervenience will assume not only the non-entailment of strong by global supervenience, but also that $(\mathrm{P})$ holds. Therefore, there is an important sense in which the utility of global supervenience depends on the truth of $(\mathrm{P})$.

Paull and Sider provide their proof of $(\mathrm{P})$ by means of this example: let $\mathrm{B}$ be a set containing just two properties, $\mathrm{P}$ and $\mathrm{Q}$; and let $\mathrm{A}$ be a set containing only $\mathrm{M}$, a property defined as follows: $\mathrm{M}(\mathrm{x})$ iff $\mathrm{P}(\mathrm{x}) \wedge \exists \mathrm{y} \mathrm{Q}(\mathrm{y})$ ( $\mathrm{x}$ has $\mathrm{M}$ just in case it has $\mathrm{P}$ and some object has Q). It is easy to see that $\mathrm{A}$ globally supervenes on $\mathrm{B}$. And for the failure of their strong supervenience consider world $\mathrm{w}$ with two individuals, a and $\mathrm{b}$ and the following distribution of properties: $\mathrm{a}$ has $\mathrm{P}$ but not $Q, b$ has $Q$ but not $P$. Let world $z$ contain only one object, c, that has $\mathrm{P}$ but not $\mathrm{Q}$. Then, a and $\mathrm{c}$ have the same B-properties but not the same A-property. ${ }^{16}$

Now the point mentioned in (ii) is this. Paull and Sider's example serves to prove $(\mathrm{P})$. But something even stronger than $(\mathrm{P})$ is usually presupposed by those who use global supervenience to assert, in a general way, a relation of dependence between families of properties. This is so because in making such claims the relata of these supposed relations of supervenience which people are interested in are not just any family of properties. In normal cases they will be kinds of properties (some natural family such as the family of physical properties) which will be put forward to constitute the set of subvenient properties for some other family. This being the case, and given what we saw in section I, interesting and normal uses of global supervenience to assert a dependence relation weaker than strong supervenience seem to presuppose something stronger than $(\mathrm{P})$, something that we could express with (S):

16 See Paull et al. (1992), pp. 840-841. 
(S) there is some family of properties A and some natural family of intrinsic and extrinsic properties B such that A supervenes globally but not strongly on B.

In Paull and Sider's example there is no guarantee that their set B does anything more than collect two arbitrary properties; no guarantee that these two properties exhaust any natural family of properties. Therefore, his proof of $(\mathrm{P})$ is not enough to prove $(\mathrm{S})$.

If (S) were false then (assuming the entailment of global supervenience by strong supervenience) a kind of equivalence thesis would hold indeed, namely thesis (E):

(E) for every natural family of intrinsic and extrinsic properties B, and every family of properties A, A supervenes strongly on $\mathrm{B}$ iff A supervenes globally on B.

Claims that were analogous to $(\mathrm{E})$ but lacked the reference to the naturality of the base set, or to the inclusion of extrinsic properties in it, would easily be shown faulty. For instance, if B is a natural family containing only intrinsic properties, we have the following counterexample to the statement that any family A globally supervenient on B is also strongly supervenient on B. Just take Paull and Sider example involved in their proof of $(\mathrm{P})$ with the following two modifications: let $\mathrm{B}$ be a natural family of intrinsic properties (e.g., the family of intrinsic physical properties), both of which are $\mathrm{P}$ and $\mathrm{Q}$; and let a and $\mathrm{c}$ share the same B-properties. Here, again, a and c would have the same B-properties but not the same A-property.

On the other hand, just to include extrinsic properties on the base set B is not sufficient either for the equivalence between global and strong supervenience. This fact is obvious from the Paull and Sider's example since in it no restriction is placed on $\mathrm{P}$ and $\mathrm{Q}$, and consequently they can perfectly be extrinsic properties. The thesis of equiva- 
lence begins to seem a plausible conjecture (echoing Kim's words quoted at the end of our section I) when we require the natural closure of the base set B (natural closure in the sense that if, e.g., $\mathrm{P}$ and $\mathrm{Q}$ belong to $\mathrm{B}$ then the rest of properties belonging to some natural set to which $\mathrm{P}$ and $\mathrm{Q}$ belong also belong to $\mathrm{B}$ ).

In fact, what Kim notes in the sentence quoted from Kim (1993) is the plausibility of the equivalence if the base set is closed under the operations of conjunction, disjunction, complementation, identity and quantification. ${ }^{17}$ Given this condition Paull and Sider's example is not a counterexample anymore, since the very property $\mathrm{M}$ belongs to any set of properties containing $\mathrm{P}$ and $\mathrm{Q}$ and subject to such condition of closure. By appealing to the naturality or natural closure of the base set, in (E), I want to achieve essentially the same result, but in a way which makes regarding that restriction on the base set as implicitly assumed in ordinary uses of thesis about supervenience (uses where the sets of properties are commonly given by comprehension, not by mere enumeration of its members) a little more plausible.

To recapitulate, global supervenience is regarded by some philosophers as a useful concept, and a concept that is different from strong supervenience. The minimum requirement for this role is that global and strong supervenience are not equivalent. They are not; Petrie, among others, has proved it. But real uses of the concept seem to require more; they seem to require at least $(\mathrm{P})$, which Paull and Sider have shown to be true. I think global supervenience would be an even more interesting and useful notion if something like (S) were also true.

17 See Kim (1993), pp. 169-170 and Kim (1987), pp. 83-85. 


\section{REFERENCES}

Burge, T., 1979, "Individualism and the Mental", Midwest Studies in Philosophy, no. 4, pp. 73-121.

García-Carpintero, M., 1994, "Dretske on the Causal Efficacy of Meaning", Mind and Language, no. 9, pp. 181-202.

Kim, J., 1993, Supervenience and Mind, Cambridge University Press, Cambridge.

— 1993, "Postscripts on Supervenience", in Supervenience and Mind, pp. 161-171.

_, 1987, "Strong' and 'Global' Supervenience Revisited", in Supervenience and Mind, pp. 79-91.

_ 1984, "Concepts of Supervenience", in Supervenience and Mind, pp. 55-78.

Lewis, D., 1983, "New Work For a Theory of Universals", Australasian Journal of Philosophy, no. 61, pp. 343-377.

McLaughlin, B.P., 1995, "Varieties of Supervenience", in E.E. Savellos and D.Ü. Yalçin (eds.), Supervenience: new essays, Cambridge University Press, Cambridge, pp. 16-59.

Paull, R.C. and T.R. Sider, 1992, "In Defense of Global Supervenience", Philosophy and Phenomenological Research, no. 52, pp. 833-854.

Petrie, B., 1987, "Global Supervenience and Reduction", Philosophy and Phenomenological Research, no. 48, pp. 119-130.

Putnam, H., 1975, "The Meaning of "Meaning" ", in Mind, Language and Reality, Cambridge University Press, Cambridge, pp. 215-271. 


\section{RESUMEN}

Mediante el concepto de superveniencia global se pretende capturar una cierta relación de dependencia entre familias de propiedades que resulte más débil que la relación de superveniencia fuerte. Sin embargo, J. Kim, el filósofo que más ha contribuido a elucidar las diferentes nociones de superveniencia, ha sugerido que cuando entre las propiedades subvenientes incluimos propiedades extrínsecas la superveniencia global resulta ser prácticamente equivalente a la superveniencia fuerte. En la primera parte de este artículo concuerdo con Kim respecto a ese punto y explico por qué si se prescinde de esa referencia a propiedades extrínsecas el concepto de superveniencia global no es apto para recoger la idea de determinación por el contexto, es decir, no es apto para cumplir con el propósito con que fue concebido. A pesar de todo, ambas relaciones de superveniencia no son estricta y lógicamente equivalentes. Por ello es importante mostrar en qué sentido son equiparables. A ello está dedicada la segunda parte del artículo, en la cual se pone en duda la relevancia de los contraejemplos presentados por Petrie y Paull y Sider a la tesis de que superveniencia global implica superveniencia fuerte. 